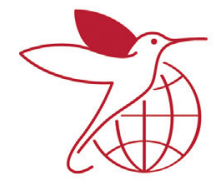

International

Diabetes

Federation

\title{
Insulin-deficient diabetes-induced bone microarchitecture alterations are associated with a decrease in the osteogenic potential of bone marrow progenitor cells: Preventive effects of metformin
}

\author{
María José Tolosa, Sara Rocío Chuguransky, Claudia Sedlinsky, \\ León Schurman, Antonio Desmond McCarthy, María Silvina Molinuevo, \\ Ana María Cortizo*
}

LIOMM (Laboratorio de Investigación en Osteopatías y Metabolismo Mineral), Department of Biological Sciences, School of Exact Sciences, National University of La Plata, La Plata, Argentina

\section{A R T I C L E I N F O}

Article history:

Received 14 March 2013

Received in revised form

21 May 2013

Accepted 30 May 2013

Available online 24 June 2013

\section{Keywords:}

Diabetes mellitus

Metformin

Bone microarchitecture

Bone marrow progenitor cells

\begin{abstract}
A B S T R A C T
Aims: Diabetes mellitus is associated with metabolic bone disease and increased lowimpact fractures. The insulin-sensitizer metformin possesses in vitro, in vivo and ex vivo osteogenic effects, although this has not been adequately studied in the context of diabetes. We evaluated the effect of insulin-deficient diabetes and/or metformin on bone microarchitecture, on osteogenic potential of bone marrow progenitor cells (BMPC) and possible mechanisms involved.

Methods: Partially insulin-deficient diabetes was induced in rats by nicotinamide/streptozotocin-injection, with or without oral metformin treatment. Femoral metaphysis microarchitecture, ex vivo osteogenic potential of BMPC, and BMPC expression of Runx-2, PPAR $\gamma$ and receptor for advanced glycation endproducts (RAGE) were investigated.

Results: Histomorphometric analysis of diabetic femoral metaphysis demonstrated a slight decrease in trabecular area and a significant reduction in osteocyte density, growth plate height and TRAP (tartrate-resistant acid phosphatase) activity in the primary spongiosa. BMPC obtained from diabetic animals showed a reduction in Runx-2/PPAR $\gamma$ ratio and in their osteogenic potential, and an increase in RAGE expression. Metformin treatment prevented the diabetes-induced alterations in bone micro-architecture and BMPC osteogenic potential. Conclusion: Partially insulin-deficient diabetes induces deleterious effects on long-bone micro-architecture that are associated with a decrease in BMPC osteogenic potential, which could be mediated by a decrease in their Runx-2/PPAR $\gamma$ ratio and up-regulation of RAGE. These diabetes-induced alterations can be totally or partially prevented by oral administration of metformin.
\end{abstract}

(C) 2013 Elsevier Ireland Ltd. All rights reserved.

\footnotetext{
* Corresponding author at: LIOMM, Department of Biological Sciences, School of Exact Sciences, National University of La Plata, 47 y 115 (1900) La Plata, Argentina. Tel.: +54 221 4235333x33; fax: +54 2214512426.

E-mail address: cortizo@biol.unlp.edu.ar (A.M. Cortizo).
} 


\section{Introduction}

In recent years there has been a growing interest in the association between diabetes and alterations in bone metabolism, which has given rise to substantial research on the effects of diabetes and of anti-diabetic drug intake on bone health [1]. Type 1 diabetes has been clearly associated with low bone mass and a 6-fold increase in low-impact fractures. On the other hand, the effects of type 2 diabetes on bone are less clear-cut: whereas bone mineral density has been reported to be either unchanged or modestly increased, incidence of low-stress fractures is almost doubled, pointing to a decrease in the bone quality of these patients [2-4]. Additionally, in both types of diabetes there is delayed fracture healing [5]. Diabetes-associated alterations in bone quality and repair have been related to poor glycemic control, to an increase in reactive oxygen species (ROS) production, and to bone matrix accumulation of advanced glycation endproducts (AGEs) [6-8]. In spite of all these reports, the underlying mechanisms of diabetes-induced bone alterations are incompletely known.

AGEs accumulation in the extracellular matrix alters bone mechanical properties through the excessive formation of nonenzymatic crosslinks between collagen chains, thus generating irreversible intra- and inter-molecular covalent bonds [9]. Additionally, collagen AGEs accumulation also induces alterations in bone cell homeostasis, mainly due to the specific recognition of AGEs by receptors such as RAGE that in osteoblasts activate intracellular signaling pathways leading to a decrease in free IGF-1 levels, and to a reduction in osteoblastic proliferation, differentiation, mineralization and survival [10-15]. Interestingly, AGEs recognition by RAGE up-regulates the expression of this receptor, thus providing a positive feedback loop that can increase the osteoblastic response to extracellular AGEs accumulation [16-18].

Osteoblasts and adipocytes derive through divergent and mutually inhibitory processes, from a common pluripotent precursor: the bone marrow progenitor cell (BMPC) of mesenchymal origin. BMPC osteoblastic differentiation is induced by the transcription factor Runx2 [19], while adipocytic differentiation is controlled by peroxisome proliferatoractivated receptor- $\gamma$ (PPAR $\gamma$ ) [20]. Rodent models of type 1 diabetes show an increase in bone marrow adiposity and PPAR $y$ activity, and a decrease in osteoblastic density with low bone mass, suggesting that their bone phenotype could be the consequence of an increase in the expression of PPAR $y$ by BMPC [21]. In type 2 diabetes, BMPC phenotypic commitment to the osteoblastic/adipocytic lineage has not been established to date.

The possible effects on bone metabolism of insulinsensitizing agents have also been studied. Thiazolidinediones induce BMPC adipogenesis and thus bone marrow adiposity, by binding to PPAR 2 receptors and enhancing expression of the adipogenic transcription factor aP2 [20,22]. Treatment of mice with thiazolidinediones decreases bone mineral content, bone formation, and trabecular bone volume, by decreasing Runx2 expression [22,23]. On the other hand, we have previously demonstrated that the biguanide metformin, widely used for treatment of type 2 diabetes, has osteogenic effects. In vitro, metformin can directly stimulate the proliferation, differentiation and mineralization of osteoblasts [24]. Metformin can also prevent the deleterious effects of AGEs on osteoblasts in culture, in part by blocking the AGEsinduced up-regulation of RAGE [17]. Oral administration of metformin to non-diabetic animals stimulates the osteogenic potential of BMPC and improves bone healing, by increasing the expression of Runx2 [25]. Due to its antioxidant and insulin-sparing effects, as well as its ability to improve cardiovascular outcomes, metformin is presently under evaluation as an adjunct therapy in patients with type 1 diabetes mellitus [26].

Based on these considerations, we hypothesized that diabetes-induced deleterious effects on bone metabolism, particularly alterations in long bone micro-architecture, could be partly due to a decrease in the osteogenic potential of BMPC; and that these alterations could be prevented by metformin treatment. To prove our hypothesis, we evaluated the effect of partially insulin-deficient diabetes and/or oral metformin administration in rats, on femoral metaphysis micro-architecture and BMPC osteogenic potential. We also studied possible mechanisms of action, such as expression of RAGE, Runx-2/PPAR $\gamma$ ratio, and pro-inflammatory cytokine levels.

\section{Materials and methods}

\subsubsection{Animal treatments}

Two-month-old male Sprague-Dawley rats (190-210 g) were used. Animals were maintained in a temperature-controlled room at $23^{\circ} \mathrm{C}$, with a fixed $12 \mathrm{~h}$ light: $12 \mathrm{~h}$ darkness cycle, and fed standard rat laboratory chow and water ad libitum. All experiments on animals were done in conformity with the Guidelines on Handling and Training of Laboratory Animals published by the Universities Federation for Animals Welfare [27]. Approval for animal studies was obtained from the institutional accreditation committee (INIBIOLP's Animal Welfare Assurance No A5647-01). In half the animals, partially insulin-deficient diabetes mellitus was induced by i.p. injection of nicotinamide (NA) $(50 \mathrm{mg} / \mathrm{kg})$ in physiological saline followed by i.p. streptozotocin (STZ) $(60 \mathrm{mg} / \mathrm{kg})$ freshly dissolved in citrate buffer $(0.05 \mathrm{M}, \mathrm{pH} 4.5)$ [28]. One week later, blood glucose was assayed to verify diabetes in all NASTZ-treated animals. Animals were then divided into four groups of 10 animals per group: control (non-treated nondiabetic) (C) and diabetic rats (D) received water ad libitum; metformin-treated non-diabetic $(M)$ and diabetic rats (D-M) received $100 \mathrm{mg} / \mathrm{kg} /$ day of metformin (Química Montpellier, Buenos Aires, Argentina) in drinking water for 2 weeks. After all treatments non-fasting blood samples were taken, and serum was stored at $-20^{\circ} \mathrm{C}$ until biochemical evaluation. Serum glucose and triglycerides were measured by commercial kits (Wiener Laboratories, Argentina), insulin by a ratspecific ELISA kit from ALPCO and fructosamine by a colorimetric BioSystems kit. Serum pro-inflammatory status was assessed by evaluation of plasmatic TNF $\alpha$ by an ELISA kit (BD OptEIA ${ }^{\mathrm{TM}}$ mouse TNF (Mono/Mono) as we have previously described [29]. 


\subsection{Histological examination of long bones}

After all treatments rats were sacrificed under anesthesia by neck dislocation, and femora were processed for histological and quantitative histomorphometric analysis. Bones were fixed in $10 \%$ formalin and decalcified in 10\% EDTA, embedded in paraffin and $5 \mu \mathrm{m}$ sections were obtained with an SM 2000R Leica microtome. The sections were stained with hematoxylin-eosin (H-E); tartrate-resistant acid phosphatase histochemistry (TRAP) (Sigma, Buenos Aires Argentina) to specifically identify osteoclasts; or Alcian Blue (AB) to evaluate the growth cartilage [25]. Pictures were taken with a Nikon Coolpix 4500 digital camera on an Eclipse E400 Nikon microscope. Images were analyzed using the Image J program (www.macbiophotonics.ca/imagej) with a Microscope scale plugin. In all experimental groups, microarchitecture of femoral metaphysis (trabecular volume and osteocytic density) was evaluated by $\mathrm{H}-\mathrm{E}$, and the average heights of femoral growth plates was determined by $\mathrm{AB}$. Osteoclastic density in the primary spongiosa was calculated as the positive TRAP area per square millimeter $\left(\mathrm{Oc} / \mathrm{mm}^{2}\right)$ [25].

\subsection{Bone marrow progenitor cell (BMPC) isolation and incubations}

BMPC were obtained from all experimental groups as previously described [25]. Briefly, bone marrow cells were collected by flushing femora and tibiae of the animals with Dulbecco's modified essential medium (DMEM) (Invitrogen, Buenos Aires, Argentina) under sterile conditions. The obtained suspension was seeded in a $25 \mathrm{~cm}^{2}$ tissue culture flask and incubated in DMEM supplemented with penicillin (100 UI/ml), streptomycin $(100 \mu \mathrm{g} / \mathrm{ml})$ and $10 \%$ fetal bovine serum (FBS) (Natocor, Córdoba, Argentina) at $37{ }^{\circ} \mathrm{C}$ in a humidified atmosphere with $5 \% \mathrm{CO}_{2}$ and 95\% air. Non-adherent cells were removed by changing the medium after $24 \mathrm{~h}$. The culture medium was changed twice a week. When cells reached confluence, the cell monolayer was detached using $0.12 \%$ trypsin-1 mM EDTA and sub-cultured in tissue culture plates.

\subsection{Osteogenic differentiation of BMPC}

BMPC were plated at a density of $5 \times 10^{4}$ cells/well in 24-well plates with $10 \%$ FBS-DMEM and incubated at $37^{\circ} \mathrm{C}$. After cells reached confluence, they were induced to differentiate into osteoblasts using an osteogenic medium (DMEM-10\% FBS containing $25 \mu \mathrm{g} / \mathrm{ml}$ ascorbic acid and $5 \mathrm{mM}$ sodium $\beta$ glycerol-phosphate) for a further 15 or 21 days [25]. Medium was changed twice a week. To evaluate alkaline phosphatase (ALP) activity, cell monolayers were washed with phosphate buffered saline (PBS) and lysed with $0.1 \%$ Triton-X100. An aliquot was used to determine ALP activity by hydrolysis of $\mathrm{p}$ nitrophenylphosphate ( $\mathrm{p}$-NPP) into $\mathrm{p}$-nitrophenol (p-NP) at $37^{\circ} \mathrm{C}$ for $1 \mathrm{~h}$, recording the absorbance at $405 \mathrm{~nm}$. Other aliquots were used for protein determination by Bradford's technique [30]. Type I collagen production was measured as previously reported [25]. Briefly, cell monolayers were fixed with Bouin's solution and stained with Sirius red dye for $1 \mathrm{~h}$. The stained material was dissolved in $1 \mathrm{ml}$ of $0.1 \mathrm{~N}$ sodium hydroxide and the absorbance of the solution was recorded at
$550 \mathrm{~nm}$. Extracellular calcium deposits (mineralization nodules) were also evaluated using Alizarin $\mathrm{S}$ red staining [25]. Stained calcium deposits were extracted with $1 \mathrm{ml}$ of $0.1 \mathrm{~N}$ sodium hydroxide, recording absorbance at $548 \mathrm{~nm}$. Alternatively, stained cultures were observed using a Nikon microscope and photographed.

\subsection{Western blot analysis}

BMPC were grown to confluence in 6-well plates in DMEM-10\% FBS and differentiated for 15 days to osteoblasts as described above. At the end of the culture periods, cells were lysed in Laemmli's buffer [31], heated to $100{ }^{\circ} \mathrm{C}$ for $3 \mathrm{~min}$, and subjected to $12 \%$ SDS-PAGE. The separated proteins were then transferred to PVDF membranes. After washing and blocking, the membranes were incubated overnight at $4{ }^{\circ} \mathrm{C}$ with an antibody directed against the osteoblastogenic transcription factor Runx-2 (Santa Cruz Biotechnology, Santa Cruz, CA, USA), or the adipogenic factor PPAR $\gamma$ (Santa Cruz Biotechnology, Santa Cruz, CA, USA). In order to normalize results, all blots were stripped and re-probed with an anti $\beta$-actin antibody (Sigma, St. Louis, MO, USA) [25]. In addition, RAGE expression was assessed with an anti-RAGE antibody (Santa Cruz Biotechnology, Santa Cruz, CA, USA) [17]. Blots were developed by an enhanced chemiluminescence method. The intensity of the specific bands was quantified by densitometry after scanning of the photographic film. Images were analyzed using the Scion-beta 2 program.

\subsection{Statistical analysis}

Results are expressed as the mean \pm SEM and were obtained from three separate experiments. Differences between the groups were assessed by one-way ANOVA using the Tukey post hoc test. For non - normal distributed data, the non parametrical Kruskal-Wallis test with Dunn post hoc test was performed, using GraphPad In Stat, version 3.00 (Graph Pad Software, San Diego, CA, USA). $\mathrm{P}<0.05$ was considered significant for all statistical analyses.

\section{Results}

\subsubsection{Biochemical evaluation of partially insulin-deficient diabetic rats}

For these studies we have used a model of partially insulindeficient diabetes [28] that is induced in rats by consecutive i.p. injection of nicotinamide and streptozotocin. After one week, diabetic and non-diabetic animals were either not treated (groups $C$ and D) or treated with metformin (groups $M$ and DM) for an additional 2 weeks. Table 1 shows the non-fasting serum biochemical profile and levels of pro-inflammatory cytokines of all groups. Significantly elevated levels of serum glucose (about 3-fold of C animals), triglycerides (4-fold of $\mathrm{C}$ ), fructosamine (1.6-fold of C) and TNF $\alpha$ (5-fold of C) were detected in D animals, while insulin was decreased (36\% of C). These parameters are compatible with a diabetic state associated with a partial destruction of pancreatic beta cells, and the consecutive induction of an inflammatory condition. 
Table 1 - Biochemical parameters and TNF- $\alpha$ levels in non-fasting plasma from animals of all experimental groups.

\begin{tabular}{|c|c|c|c|c|}
\hline Parameter & C & M & $\mathrm{D}$ & D-M \\
\hline Glucose [mg/dL] & $165 \pm 6$ & $181 \pm 9$ & $453 \pm 40^{*}, \#$ & $309 \pm 19^{*}, \# \#, \&$ \\
\hline Insulin $[\mathrm{ng} / \mathrm{mL}]$ & $1.26 \pm 0.17$ & $1.09 \pm 0.30$ & $0.45 \pm .06^{* * *}$ & $0.37 \pm 0.12^{* * * \# \#}$ \\
\hline Triglycerides [mg/dL] & $62 \pm 7$ & $54 \pm 4$ & $252 \pm 25^{*}, \#$ & $128 \pm 19^{* * * * \# \#, 8}$ \\
\hline Fructosamine $[\mu \mathrm{mol} / \mathrm{L}]$ & $147 \pm 26$ & $190 \pm 13$ & $239 \pm 12^{* *}$ & $236 \pm 16^{* *}$ \\
\hline $\mathrm{TNF}-\alpha[\mathrm{pg} / \mathrm{mL}]$ & $24.7 \pm 1.8$ & $22.4 \pm 2.7$ & $123 \pm 9.0^{*}, \#$ & $96.9 \pm 8.6^{*}, \#, \& \&$ \\
\hline $\begin{array}{l}\text { Results are expressed as } \\
\text { Difference from C: } \mathrm{P}< \\
\text { Difference from C: } \mathrm{P}< \\
\text { Difference from C: } \mathrm{P} \\
\# \text { Differences from M: } P \\
\text { \#\# Differences from M: } P \\
\text { \& Differences from D: } P \\
\text { \&\& Differences from D: }\end{array}$ & $n=10$ & & & \\
\hline
\end{tabular}

Metformin treatment (DM group) partially prevented the hyperglycemia (about 2-fold of C) and hypertriglyceridemia (2-fold of $\mathrm{C}$ ) associated with diabetes, although the animals still showed poor metabolic control and inflammatory stress, as evidenced by the high levels of fructosamine and TNF $\alpha$ (4fold of $\mathrm{C}$ ). In non-diabetic animals, metformin did not affect any biochemical parameters (M group).

\subsection{Diabetes-induced alterations in femoral micro- architecture are prevented by metformin treatment}

To assess the impact of partially insulin-deficient diabetes on bone microarchitecture, we performed a histomorphometric analysis of long bones. In femoral metaphysis $2 \mathrm{~mm}$ distal from the cartilage growth plate, $5 \mu \mathrm{m}$ decalcified bone sections were stained with $\mathrm{H}-\mathrm{E}$ to evaluate relative trabecular area and osteocyte density. In addition, TRAP staining was performed to evaluate the proportion of osteoclast-covered bone surface in the primary spongiosa. Metaphyseal bone marrow adiposity was also evaluated in the stained sections (see Fig. 1). Growth plate morphometry was evaluated after Alcian Blue staining (Fig. 1I-L). Analysis of samples from all experimental groups revealed changes in trabecular bone associated with diabetes and/or metformin treatment (Table 2). Compared with control rats, diabetic rats exhibited a slight although not statistically significant decrease in trabecular area (85\%), along with a significant decrease in osteocyte density (60\%) (Fig. 1A-D), TRAP activity (36\%) (Fig. 1E-H) and growth plate height (47\%) (Fig. 1I-L). A significant increase in femoral metaphysis bone marrow adiposity was detected in diabetic animals (Fig. $1 \mathrm{G}$ and $\mathrm{K}$ ). Metformin treatment of non-diabetic animals induced a significant increase in femoral osteocyte density (163\% of C) (Fig. 1B) and TRAP activity (216\% of C) (Fig. 1F). In diabetic animals, metformin treatment partially prevented the diabetesinduced decrease in osteocyte density (Fig. 1D), TRAP activity (Fig. 1H) and growth plate height (Fig. 1L), and completely prevented the increase in bone marrow adiposity (Fig. $1 \mathrm{H}$ and L).

\subsection{Partially insulin-deficient diabetes impairs the osteogenic commitment of rat BMPC: effect of in vivo metformin treatment}

We evaluated the effects of a pharmacological induction of diabetes and the consecutive in vivo treatment with metformin, on the osteogenic potential of BMPC in vitro. Cells isolated from animals of each experimental group were cultured until confluence and then induced to differentiate in the presence of beta-glycero-phosphate and ascorbic acid during 15 or 21 days. Type I collagen production and alkaline phosphatase activity were used as markers of osteoblastic differentiation. Fig. 1 shows that after 15 days of differentiation, BMPC from $D$ rats exhibited a significant decrease in collagen production (Fig. 2A) and ALP activity (Fig. 2B). In vivo treatment with metformin ( $D-M$ group) partially or totally abolished these diabetes-induced alterations in BMPC osteogenic capacity. In addition, we confirmed our previous observation [25] that BMPC from non-diabetic metformintreated rats $(\mathrm{M})$ expressed higher levels of ALP than BMPC from control rats, and showed a significant increase in type I collagen production. Similarly after 21 days of differentiation, diabetes decreased the mineralizing capacity of BMPC cultures (Fig. 2C). In vivo metformin treatment increased the mineralizing capacity of BMPC from non-diabetic rats ( $M$ group), and completely prevented the diabetes-induced decrease in mineralization of BMPC from diabetic rats (D-M group).

\subsection{Effect of diabetes and metformin on osteoblastic and adipogenic transcription factors}

We next evaluated possible changes in the expression of the two major transcription factors involved in osteogenic and adipogenic differentiation, Runx2 and PPAR $\gamma$. BMPC from each experimental group were cultured in an osteogenic media for 15 days and the transcription factors evaluated by Western immunoblot. Fig. 3A shows that BMPC from D rats expressed significantly lower levels of Runx2 than C-derived BMPC. In addition, BMPC from metformin-treated nondiabetic rats expressed similar levels of Runx2 to control BMPC, whereas metformin treatment in diabetic rats partially prevented the diabetes-induced decrease in Runx2 expression of BMPC.

Analysis of the expression of PPAR $\gamma$ showed that this adipogenic factor was significantly increased in BMPC obtained from diabetic rats, compared to control BMPC, after 15 days of osteogenic induction (Fig. 3B). In vivo treatment of diabetic and non-diabetic rats with metformin induced in both cases a decrease in the expression of PPARy by BMPC. 

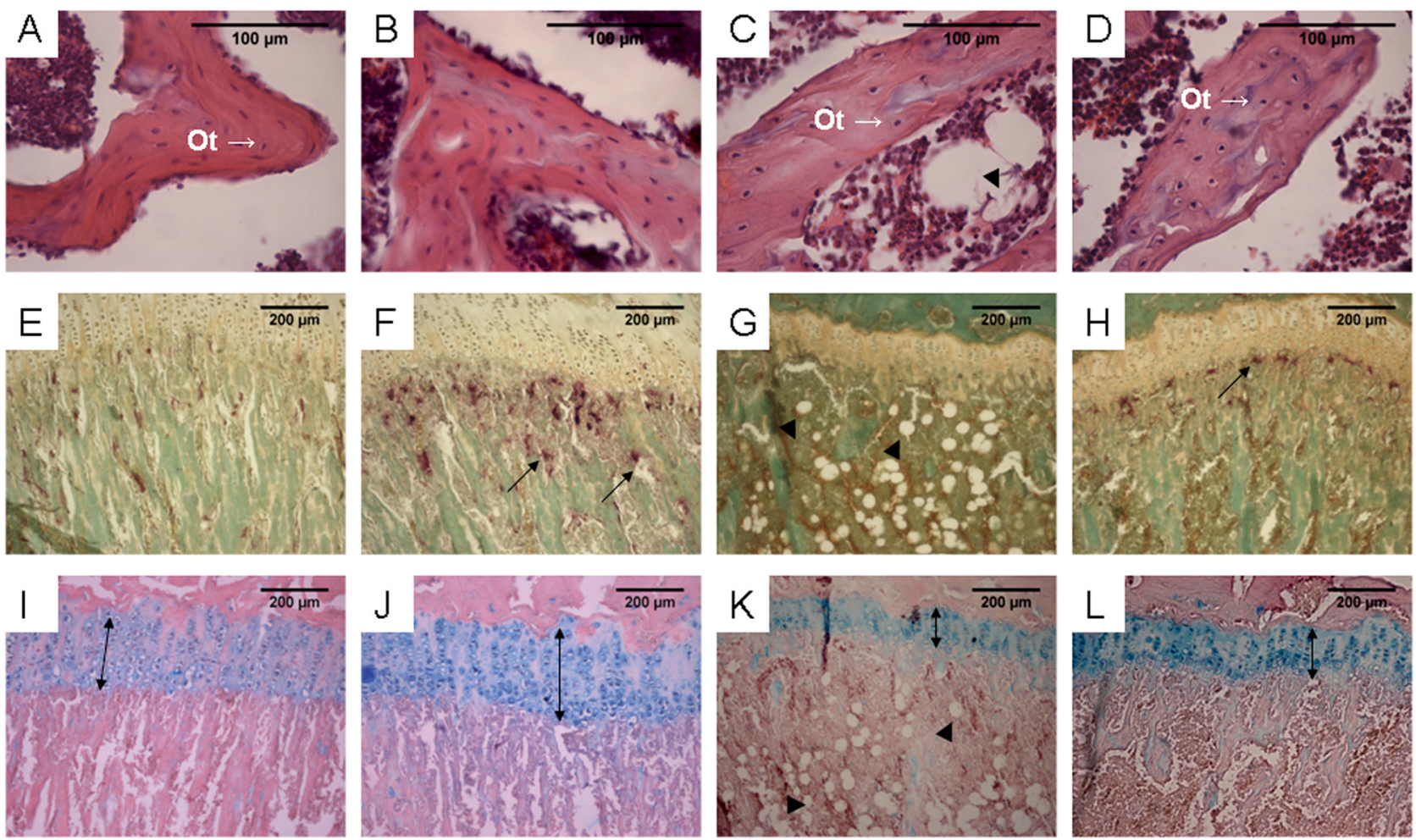

Fig. 1 - Effect of metformin on diabetes-induced microarchitecture alterations of rat femur. Representative histological photos of proximal femur from control (A, E, I), metformin-treated non-diabetic (100 mg/kg/day) (B, F, J), untreated diabetic (C, G, K) and metformin-treated diabetic (D, H, L) rats. After 15 days, all animals were sacrificed, and the femora were examined by histological procedures. Bone sections were stained with hematoxiline-eosin (A-D), analyzed for TRAPactivity in the primary spongiosa (E-H) or stained with Alcian Blue (I-L) to assess height of the growth plate. Note the reduced TRAP-activity (arrow) accompanied by thin growth plate height $(\leftrightarrow)$ and high adiposity (arrowhead) in samples from untreated diabetic versus non-diabetic animals. In contrast, increased TRAP-activity (H), no adiposity (H and L) and a trend to normalize the growth plate height $(\mathrm{L})$ are observed in the samples of metformin-treated diabetic animals.

3.5. Diabetes and metformin regulate the expression of RAGE by BMPC

We next investigated the expression of the receptor RAGE in BMPC obtained from all experimental groups, prior to and after 15 days of osteogenic differentiation. RAGE was evaluated in cell lysates by Western immunoblot. Fig. 4 shows that BMPC obtained from group $C$ expressed low levels of RAGE. The expression of RAGE was significantly increased in BMPC from group $\mathrm{D}$, both prior to and after 15 days of osteogenic differentiation. In vivo metformin treatment did not modify RAGE expression in BMPC from non-diabetic animals

Table 2 - Histomorphometric analysis of tissue sections from animals of all experimental groups.

\begin{tabular}{|c|c|c|c|c|}
\hline Parameter & C & M & $\mathrm{D}$ & D-M \\
\hline Tb.Ar/T.Ar [\%] & $39 \pm 2$ & $45 \pm 3$ & $33 \pm 2^{\#}$ & $41 \pm 3$ \\
\hline N.Ot $\left[\# / \mathrm{mm}^{2}\right]$ & $1515 \pm 148$ & $2477 \pm 223^{* * *}$ & $898 \pm 103^{*}, \# \#$ & $1470 \pm 108^{* *, \# \#, \& 8}$ \\
\hline TRAP.Ar [\%] & $6.1 \pm 0.7$ & $13.2 \pm 2.1^{* * *}$ & $2.2 \pm 0.5^{* * *}, \# \#$ & $8.2 \pm 0.9^{*}, \#, \& \& \&$ \\
\hline Growth plate height $[\mu \mathrm{m}]$ & $210 \pm 3$ & $226 \pm 8$ & $103 \pm 5^{* \cdots, \# \#}$ & $128 \pm 4^{* * *, \# \#, \& \& 8}$ \\
\hline \multicolumn{5}{|c|}{$\begin{array}{l}\text { Tb.Ar/T.Ar, relative trabecular area to tissue area. N.Ot, Osteocyte number. TRAP.Ar, relative TRAP area to bone area. } \\
\text { Differences from C: } P<0.05 \text {. } \\
\text { Differences from C: } P<0.01 \\
\text { Differences from C: } P<0.001 \\
\text { \# Differences from } \mathrm{M}: P<0.05 \\
\text { \# Differences from } \mathrm{M}: \mathrm{P}<0.001 \\
\text { \& Differences from } \mathrm{D}: \mathrm{P}<0.05 \\
\text { \&\& Differences from } \mathrm{D}: \mathrm{P}<0.01 \\
\text { \&\&\& Differences from } \mathrm{D}: \mathrm{P}<0.001\end{array}$} \\
\hline
\end{tabular}



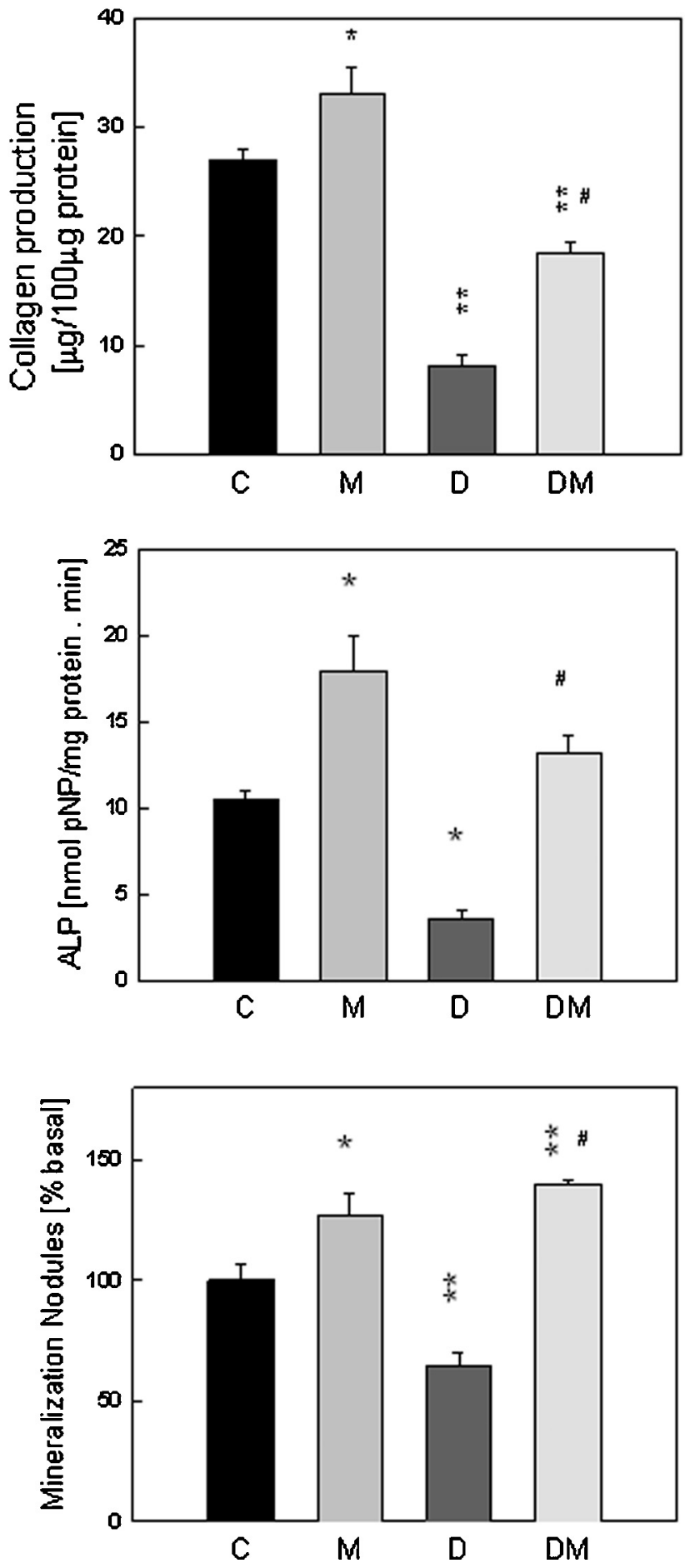

Fig. 2 - In vivo oral administration of metformin in diabetic and non-diabetic rats reduces the in vitro osteogenic potential of BMPC. BMPC were isolated from control (C), metformin-treated non-diabetic (100 mg/kg/day) (M), untreated diabetic (D) and metformin-treated diabetic (DM) rats. After confluence, cells were differentiated in an osteogenic media for 15 days (A,B) or 21 days (C). After these culture periods, type 1 collagen production (A), ALP activity (B) and mineralizing capacity (C) were evaluated. Values are expressed as mean \pm SEM. Differences from C: ${ }^{*} \mathrm{P}<0.01,{ }^{* *} \mathrm{P}<0.001$; differences vs. $\mathrm{D}$ : ${ }^{\#} \mathrm{P}<0.001$.
A
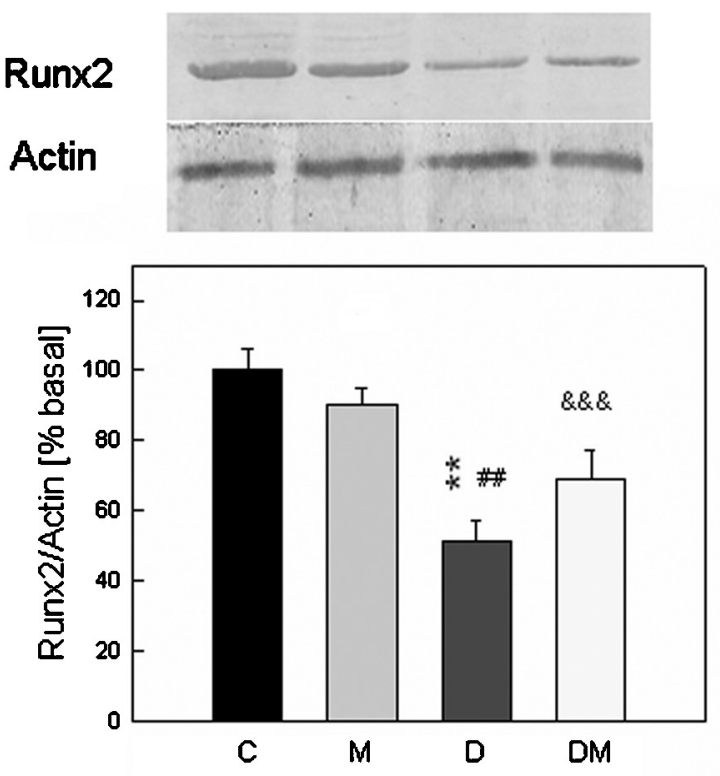

8

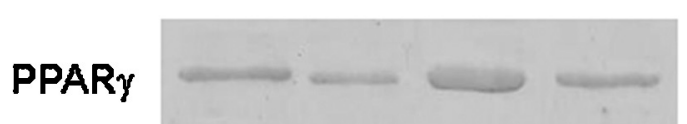

Actin

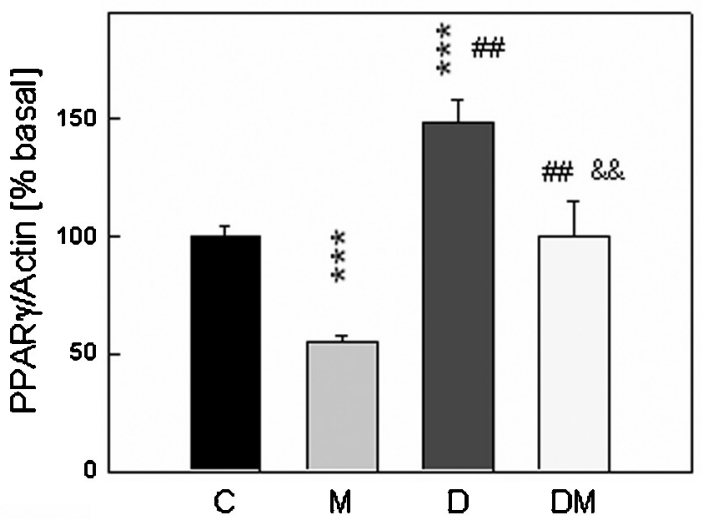

Fig. 3 - Induction of Diabetes and oral administration of metformin alter the expression of Runx2 and PPAR $\gamma$ in BMPC. BMPC were isolated from control (C), metformintreated non-diabetic (100 $\mathrm{mg} / \mathrm{kg} /$ day) (M), untreated diabetic (D) and metformin-treated diabetic (DM) rats. After confluence, cells were differentiated in an osteogenic media for 15 days. Expression of the osteogenic transcription factor Runx2 and house-keeping protein actin (A) or adipogenic factor PPAR $\gamma$ and actin (B) were evaluated by Western immunoblot. The blots were quantified with the Scion beta 2 image program; data were normalized against actin and expressed as percentage of $C$ data. Values are expressed as mean \pm SEM. Differences from C: ${ }^{*} \mathrm{P}<0.05,{ }^{* *} \mathrm{P}<0.01,{ }^{* * *} \mathrm{P}<0.001$; differences from $\mathrm{M}$ : ${ }^{\# \#} P<0.001$; differences vs. $D:{ }^{\& \&} P<0.01,{ }^{\& \& \&} P<0.001$. 
A

\section{RAGE}

\section{Actin}

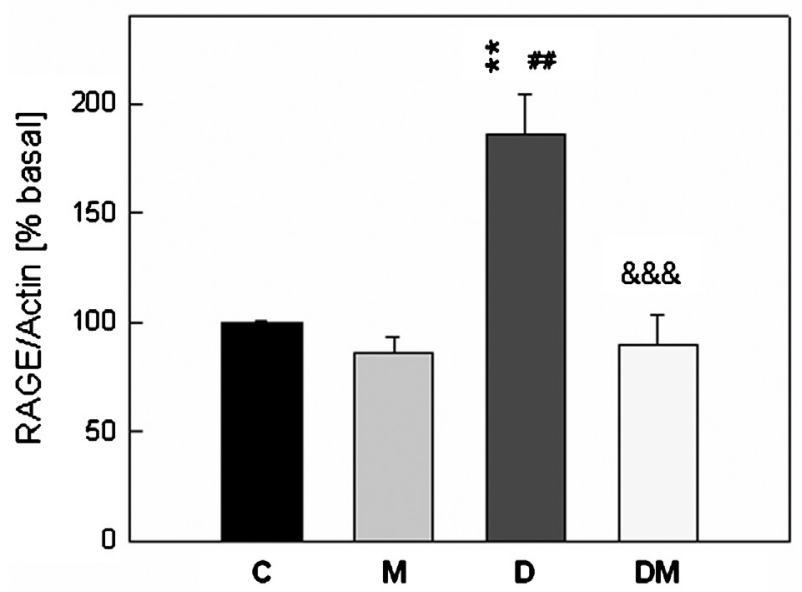

B

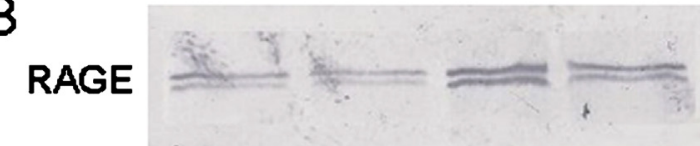

Actin

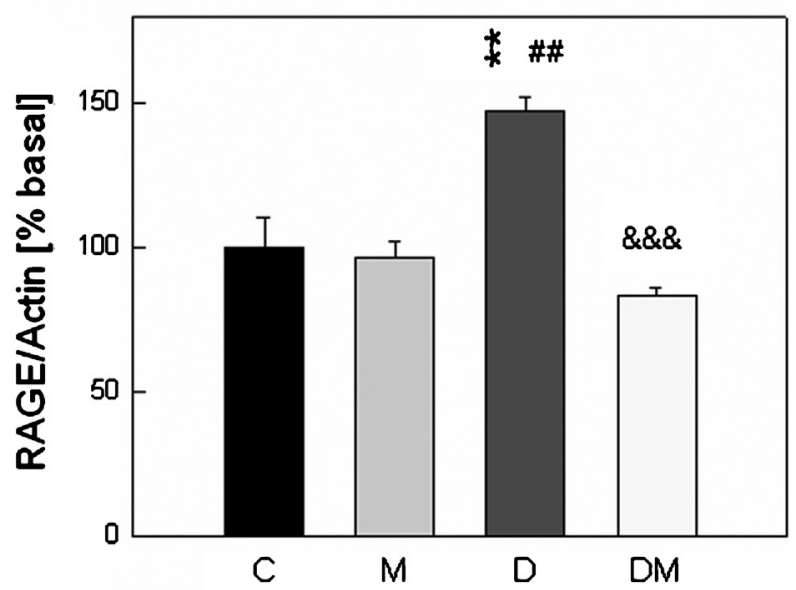

Fig. 4 - Expression of RAGE by BMPC obtained from diabetic and metformin-treated rats. BMPC were isolated from control (C), metformin-treated non-diabetic $(100 \mathrm{mg} / \mathrm{kg} /$ day) (M), untreated diabetic (D) and metformin-treated diabetic (DM) rats. After confluence, cells were lysed directly (A) or after a 15 day differentiation in osteogenic media (B). In the lysates, expression of RAGE and actin was evaluated by Western immunoblot and quantified with the Scion beta 2 image program; RAGE data were normalized against actin and expressed as percentage of $C$ data. Values are expressed as the mean \pm SEM. Differences from C: ${ }^{* *} \mathrm{P}<0.01$; differences from $\mathrm{M}$ : ${ }^{\# \#} \mathrm{P}<0.001$; differences vs. $D$ : ${ }^{\& \& \&} \mathrm{P}<0.001$. (group M). However, in vivo metformin treatment (D-M group) completely prevented the diabetes-associated up-regulation of RAGE expression in BMPC.

\section{Discussion}

Increasing clinical and experimental evidence has accumulated indicating that diabetes mellitus is associated with metabolic bone disease, osteoporosis and low-impact fractures [32]. Patients with type 1 diabetes tend to have low bone mineral density and thus an increase in osteoporotic fractures. Individuals with type 2 diabetes, although characterized by normal or elevated bone mineral density, also show a significant increase in fracture risk. The causes of diabetic bone disease have not been adequately identified, however type 2 diabetes may arise from two usually concurrent pathogenic mechanisms, insulin deficiency and insulin resistance, both of which could in theory affect bone metabolism. One possible approach to circumvent this issue is to evaluate each mechanism separately by using models of partial insulin deficiency or of insulin resistance.

Different animal models have been used to investigate the mechanisms underlying bone alterations in diabetes. Streptozotocin-induced severely insulinopenic rodents have served as a model for type 1 diabetes. Recently, a model of streptozotocin-nicotinamide-induced partially insulin-deficient diabetic rats has been described [28]. These animals are characterized by moderate hyperglycaemia, dislipidemia and impaired glucose tolerance due to the loss of early-phase insulin secretion, but not insulin resistance or obesity. Using this model, in the present study we have investigated the effect of partially insulin-deficient diabetes with or without oral metformin treatment, on the microarchitecture of long bones and on the osteogenic potential of BMPC. Diabetic animals showed a deficit in insulin secretion (without insulin resistance), hypertriglyceridemia, elevated serum pro-inflammatory cytokine levels, and increased non-enzymatic glycosylation of serum proteins. These observations are in agreement with previous reports that have used the same model [33]. Additionally, after two weeks of oral metformin treatment we found a partial compensation in the biochemical parameters of diabetic animals.

Since diabetes is clearly associated with metabolic bone disease, hyperglycaemia has been postulated to be a major factor that could directly or indirectly affect bone formation [32]. Hyperglycaemia leads to excessive non-enzymatic glycosylation of proteins with accumulation of AGEs, thus increasing AGEs-RAGE interaction as well as the induction of cytokines (TNF- $\alpha$, IL-6, HMGB1), oxidative stress and osteoclastogenic mediators. In this setting, the normal microarchitecture of bone could be affected, leading to a reduction in bone quality as has been suggested by the results of bone mechanical loading [9,34]. In addition, diabetes induces lipid accumulation in the bone marrow, and this could be due to an excessive adipogenic differentiation of BMPC, thus decreasing the availability of differentiated osteblasts for bone formation [22]. The selection of adipogenesis over osteoblastogenesis has been reported in type 1 diabetes, osteoporosis and disuse $[21,35]$. 
In our experimental insulin-deficient diabetic model, bone histomorphometric studies demonstrated a slight decrease in femoral metaphysis trabecular bone together with an important decrease in osteocyte density. We also found a dramatic increase in the adiposity of the bone marrow of diabetic animals, an effect that could further contribute to the deterioration of bone quality. Our present observations are in agreement with the histomorphometric and micro-computed tomographic analysis of bone performed by other investigators in NOD and streptozotocin-induced type 1 diabetes mice models $[34,36]$.

Additionally, in our diabetic rats we found a great reduction in TRAP activity of the primary spongiosa and in the height of the growth plate. The growth plate height depends on two antagonistic processes: one in the direction from epiphysis to metaphysis involving the production, proliferation and hypertrophy of chondrocytes; and another in the opposite direction representing vascular invasion and cartilage replacement by new bone [17]. This last process requires osteoclastic activity, and so our results of a combined reduction of TRAP activity in the primary spongiosa and of growth plate height, are compatible with a decrease in bone longitudinal growth. Using STZ-induced type 1 diabetic rats, Silva et al. [34] also found a significant decrease in the growth plate thickness although they did not evaluate TRAP activity. On the other hand, other researchers have found a diabetesinduced increase in osteoclast action, although not in growth plates and thus associated with an increase in long bone fragility and a decrease in bone healing $[37,38]$.

The osteocytic network is critical to bone health, since these cells act as mechanosensors and regulate bone remodeling [39]. Loss of osteocytes due to apoptosis as a consequence of diabetes induction has been previously reported in bone healing and in the cortical tibia of type 1 diabetic rodents $[25,40]$. It has also been postulated that apoptotic osteocytes release signals that in turn activate osteoclasts and bone resorption [41]. One of these signals is the high mobility group box 1 (HMGB1) protein, which upregulates the expression of pro-inflammatory cytokines by interacting with different receptors, including RAGE for which it is a physiological ligand. Additionally, induction of diabetesassociated oxidative stress could be one of the main insults generating osteocyte apoptosis [42]. These observations suggest that AGEs-induced ROS production and/or increased cytokine generation could be responsible for the greatly diminished osteocyte density observed in our insulin-deficient diabetic rat model.

Metformin, one the most commonly used oral antihyperglycaemic agents in individuals with type 2 diabetes, is an insulin sensitizer that has been shown to have antioxidant effects and to prevent long-term diabetic complications [43]. The use of this drug in patients with type 1 diabetes mellitus is currently being evaluated as an adjunct therapy to insulin, and as such it has been shown to reduce insulin requirement, body weight and macrovascular disease, and to improve endothelial function [44-46]. We have previously demonstrated that metformin possesses in vitro, in vivo and ex vivo osteogenic effects [24], although this has not been adequately studied in diabetic animals. Consequently, in the present study we investigated the in vivo effect of this insulin-sensitizer on bone alterations induced by a diabetic state that does not show insulin resistance. This fact is important since any effect of metformin on bone tissue would probably then be direct, and not due to the bone actions of an increase in insulin sensitivity. After 2 weeks of oral treatment, metformin was able to prevent the diabetes-induced trabecular bone loss and increase in bone marrow adiposity. Metformin also increased both trabecular osteocytic density and TRAP activity of the primary spongiosa, in diabetic and non-diabetic animals. In addition, the decrease in growth plate height of diabetic animals was partially prevented by oral treatment with metformin.

BMPC can be induced to differentiate toward different phenotypes by alterations in their micro-environment. Thus, a diabetes-induced increase in bone AGEs, cytokines and/or oxidative stress, could affect the fate of BMPC. To investigate the mechanisms by which diabetes affects bone formation, we performed a series of studies with BMPC obtained from the femora of animals from each experimental group. Our results demonstrate that the capacity for osteogenic induction of BMPC from diabetic animals is significantly decreased, as evaluated by type 1 collagen production, ALP expression and their capacity for matrix mineralization. These findings in BMPC of diabetic origin were associated both with a significant decrease in their expression of the master osteoblastogenic transcription factor Runx2, and with a significant increase in their expression of the adipogenic PPAR $\gamma$. All of these alterations in BMPC osteogenic induction were completely prevented if the diabetic animals were treated for 2 weeks with orally administered metformin. The imbalance in Runx2/ PPAR $y$ ratio that we are reporting in animals with diabetes is likely a critical event that could explain the reduced osteogenic potential of BMPC and in consequence also the bone histomorphometric alterations that we have observed in this model. A decrease in Runx2 expression has also been previously described in STZ-induced type 1 diabetic mice with marrow ablation [47]. However, other investigators found no change in the number of osteoblasts, ALP expression or Runx2 mRNA levels in type 1 diabetic mice [21]. These authors suggest that diabetic bone loss could be secondary to an increase in bone marrow adiposity. Our present data, showing an increase both in the adipogenic factor PPAR $\gamma$ and in the adiposity of the bone marrow, give support to this hypothesis.

Individuals with long-standing diabetes show an increase in their systemic accumulation of AGEs, which are then recognized by specific receptors such as RAGE expressed by various cell types. Our group and other researchers have previously demonstrated the expression of RAGE in osteoblasts and in BMPC, as well as an AGEs-induced up-regulation of RAGE in these cell types $[11,12,16,48,49]$. In vitro, metformin can prevent the AGEs-induced up-regulation of RAGE in osteoblasts in culture [17]. To evaluate possible mechanisms involved in diabetes-induced bone alterations, in the present study we investigated the expression of RAGE by BMPC obtained from animals of all groups. In our experimental conditions, diabetes induced a significant increase in BMPC RAGE expression, both prior to and after 15 days of osteogenic induction, and this increase was prevented if the diabetic animals were treated for 2 weeks with metformin. It has 
previously been demonstrated for this streptozotocin-nicotinamide diabetic model, that oxidative stress and levels of inflammatory cytokines are elevated [33]. In coincidence with those results of other authors, and possibly related to the diabetes-induced up-regulation of RAGE, we also found an increase in serum TNF $\alpha$ levels in diabetic animals that was prevented by oral treatment with metformin.

In conclusion, with our model of partial insulin deficiency we believe that we have provided sufficient evidence to adequately confirm the hypothesis of this study, and we are additionally proposing the modulation of RAGE expression by BMPC as a possible pathogenic mechanism for diabetic osteopathy. We consider that the novelty of our study consists in our ability to have provided a feasible explanation for alterations in the micro-architecture of diabetic bone: namely, by a decrease in the osteogenic potential of BMPC. Also by having demonstrated for the first time in a model of diabetes, that these alterations of bone tissue can be partially reverted by oral metformin treatment.

\section{Conflict of interest}

The authors declare that they have no conflict of interest.

\section{Acknowledgments}

We wish to thank Química Montpellier Argentina for their generous donation of metformin. This work was partially supported by grants from Universidad Nacional de La Plata, Comisión de Investigaciones Científicas de la Provincia de Buenos Aires (CICPBA), and Agencia (PICT1083). AMC is a member of the Carrera del Investigador, CICPBA. MSM is a member of the Carrera del Investigador, CONICET. ADM is a part-time professor and researcher of UNLP.

\section{R E F E R E N C E S}

[1] Pietschman P, Patsch JM, Schernataner G. Diabetes and bone. Horm Metab Res 2010;42:763-8.

[2] Janghorbani M, Feskanich D, Willett WC, Hu F. Prospective study of diabetes and risk of hip fracture The Nurses' Health Study. Diabetes Care 2006;29:1573-8.

[3] Janghorbani M, Van Dam RM, Willett WC, Hu FB. Systematic review of type 1 and type 2 diabetes mellitus and risk of fracture. Am J Epidemiol 2007;166:495-505.

[4] Thrailkill KM, Lumpkin Jr CK, Bunn RC, Kemp SF, Fowlkes JL. Is insulin an anabolic agent in bone? Dissecting the diabetic bone for clues. Am J Physiol Endocrinol Metab 2005;289:E735-45.

[5] Loder RT. The influence of diabetes mellitus on the healing of closed fractures. Clin Orthop Relat Res 1988;232:210-6.

[6] Hamann C, Goettsch C, Mettelsiefen J, Henkenjohann V, Rauner M, Hempel U, et al. Delayed bone regeneration and low bone mass in a rat model of insulin-resistant type 2 diabetes mellitus is due to impaired osteoblast function. Am J Physiol Endocrinol Metab 2011;301:E1220-8.

[7] Roszer T. Inflammation as death or life signal in diabetic fracture healing. Inflamm Res 2011;60:3-10.
[8] Santana RB, Xu L, Chase HB, Amar S, Graves DT, Trackman PC. A role for advanced glycation end products in diminished bone healing in type 1 diabetes. Diabetes 2003;52:1502-10.

[9] Saito M, Marumo K. Collagen cross-links as a determinant of bone quality: a possible explanation for bone fragility in aging, osteoporosis, and diabetes mellitus. Osteoporos Int 2010;21:195-214.

[10] Alikhani M, Alikhani Z, Boyd C, MacLellan CM, Raptis M, Liu $\mathrm{R}$, et al. Advanced glycation end products stimulate osteoblast apoptosis via the MAP kinase and cytosolic apoptotic pathways. Bone 2007;40:345-53.

[11] Cortizo AM, Lettieri MG, Barrio DA, Mercer N, Etcheverry $\mathrm{SB}$, McCarthy AD. Advanced glycation end-products (AGEs) induce concerted changes in the osteoblastic expression of their receptor RAGE and in the activation of extracellular signal-regulated kinases (ERK). Mol Cell Biochem 2003;250:1-10.

[12] Franke S, Rüster C, Pester J, Hofmann G, Oelzner P, Wolf G. Advanced glycation end products affect growth and function of osteoblasts. Clin Exp Rheumatol 2011;29:650-60.

[13] McCarthy AD, Etcheverry SB, Bruzzone L, Cortizo AM. Effects of advanced glycation end-products on the proliferation and differentiation of osteoblast-like cells. Mol Cell Biochem 1997;170:43-51.

[14] McCarthy AD, Etcheverry SB, Bruzzone L, Lettieri G, Barrio DA, Cortizo AM. Non-enzymatic glycosylation of a type I collagen matrix: effects on osteoblastic development and oxidative stress. BMC Cell Biol 2001;2:16.

[15] McCarthy AD, Etcheverry SB, Cortizo AM. Effect of advanced glycation endproducts on the secretion of insulin-like growth factor-I and its binding proteins: role in osteoblast development. Acta Diabetol 2001;38:113-22.

[16] Mercer N, Ahmed H, Etcheverry SB, Vasta GR, Cortizo AM. Regulation of advanced glycation end product (AGE) receptors and apoptosis by AGEs in osteoblast-like cells. Mol Cell Biochem 2007;306:87-94.

[17] Schurman L, McCarthy AD, Sedlinsky C, Gangoity MV, Arnol V, Bruzzone L, et al. Metformin reverts deleterious effects of advanced glycation end-products (AGEs) on osteoblastic cells. Exp Clin Endocrinol Metab 2008;116:333-40.

[18] Zhou Z, Xiong WC. RAGE and its ligands in bone metabolism. Front Biosci (Schol Ed) 2011;3:768-76.

[19] Zaidi M. Skeletal remodeling in health and disease. Nat Medicine 2007;13:791-801.

[20] Lecka-Czernik B, Moerman EJ, Grant DF, Lehmann JM, Manolagas SC, Jilka RL. Divergent effects of selective peroxisome proliferator-activated receptor $\gamma 2$ ligands on adipocyte versus osteoblast differentiation. Endocrinology 2002;143:2376-84.

[21] Botolin S, Faugere MC, Malluche H, Orth M, Meyer M, McCabe LR. Increased bone adiposity and peroxisome proliferator-activated receptor- $\gamma 2$ in type 1 diabetic mice. Endocrinology 2005;146:3622-31.

[22] Ali A, Weinstein RS, Stewart SA, Parfitt AM, Manolagas SC, Jilka RL. Rosiglitazone causes bone loss in mice by suppressing osteoblast differentiation and bone formation. Endocrinology 2005;146:1226-35.

[23] Rzonca SO, Suva LJ, Gaddy D, Montague DC, Lecka-Czernik B. Bone is a target for the antidiabetic compound rosiglitazone. Endocrinology 2004;145:401-6.

[24] Cortizo AM, Sedlinsky C, McCarthy AD, Blanco A, Schurman L. Osteogenicactions of the anti-diabetic drug metformin on osteoblasts in culture. Eur J Pharmacol 2006;536:38-46.

[25] Molinuevo MS, Schurman L, McCarthy AD, Cortizo AM, Tolosa MJ, Gangoiti MV, et al. Effect of metformin on bone 
marrow progenitor cell differentiation: in vivo and in vitro studies. J Bone Miner Res 2010;25:211-6.

[26] George P, McCrimmon RJ. Potential role of non-insulin adjunct therapy in Type 1 diabetes. Diabetic Med 2013;30:179-88.

[27] Guidelines on Handling and Training of Laboratory Animals. In: Purl UFA, editor. The Biological Council of Animal Research, Welfare Panel. Guide for the Care and Use of Laboratory Animals: Eighth Edition. Washington D.C.: The National Academies Press; 2011.

[28] Tamara A, Matsuyama-Yokono A, Nakano R, Someya Y, Shibasaki M. Hypoglycaemic effects of antidiabetic drugs in streptozotocin-nicotinamide-induced mildly diabetic and streptozotocin-induced severely diabetic rats. Basic Clin Pharmacol Toxicol 2008;103:560-8.

[29] Cortizo AM, Ruderman G, Correa G, Mogilner IG, Tolosa EJ. Effect of surface topography of collagen scaffolds on Cytotoxicity and osteoblast differentiation. J Biomater Tissue Eng 2012;2:1-8.

[30] Bradford MM. A rapid and sensitive method for the quantitation of microgram quantities of protein utilizing the principle of protein-dye binding. Anal Biochem 1976;72:248-54.

[31] Laemmli UK. Cleavage of structural protein during the assembly of the head of bacteriophage T4. Nature 1970;227:680-5.

[32] Wongdee K, Charoenphandhu N. Osteoporosis in diabetes mellitus: possible cellular and molecular mechanisms. World J Diabetes 2011;15:41-8.

[33] Arya A, Cheah SC, Looi CY, Taha H, Rais Mustafa M, Mohd MA. The methanolic fraction of centratherum anthelminticum seed downregulates pro-inflammatory cytokines, oxidative stress, and hyperglycemia in STZnicotinamide-induced type 2 diabetic rats. Food Chem Toxicol 2012;50:4209-20.

[34] Silva MJ, Brodt MD, Lynch MA, McKenzie JA, Tanouye KM, Nyman JS, et al. Type 1 diabetes in young rats leads to progressive trabecular bone loss, cessation of cortical bone growth, and diminished whole bone strength and fatigue life. J Bone Miner Res 2009;24:1618-27.

[35] Nuttall ME, Gimble JM. Controlling the balance between osteoblastogenesis and adipogenesis and the consequent therapeutic implications. Curr Opin Pharmacol 2004;4:290-4.

[36] Nyman JS, Even JL, Jo CH, Herbert EG, Murry MR, Cockrell $\mathrm{GE}$, et al. Increasing duration of type 1 diabetes perturbs the strength-structure relationship and increases brittleness of bone. Bone 2011;48:733-40.
[37] Kawashima Y, Fritton JC, Yakar S, Epstein S, Schaffler MB, Jepsen $\mathrm{KJ}$, et al. Type 2 diabetic mice demonstrate slender long bones with increased fragility secondary to increased osteoclastogenesis. Bone 2009;44:648-55.

[38] Kayal RA, Tsatsas D, Bauer MA, Allen B, Al-Sebaei MO, Kakar S, et al. Diminished bone formation during diabetic fracture healing is related to the premature resorption of cartilage associated with increased osteoclast activity. J Bone Miner Res 2007;22:560-8.

[39] Kogianni G, Noble BS. The biology of osteocytes. Curr Osteoporos Rep 2007;5:81-6.

[40] Portal-Núñez S, Lozano D, de Castro LF, de Gortázar AR, Nogués X, Esbrit P. Alterations of the Wnt/beta-catenin pathway and its target genes for the $\mathrm{N}$ - and $\mathrm{C}$-terminal domains of parathyroid hormone-related protein in bone from diabetic mice. FEBS Lett 2010;584:3095-100.

[41] Bidwell JP, Yang J, Robling AG. Is HMGB1 an osteocyte alarmin? J Cell Biochem 2008;103:1671-80.

[42] Manolagas SC. From estrogen-centric to aging and oxidative stress: a revised perspective of the pathogenesis of osteoporosis. Endocr Rev 2010;31:266-300.

[43] Wiernsperger NF. Metformin: intrinsic vasculoprotective properties. Diabetes Technol Ther 2000;2:259-72.

[44] Lund SS, Tarnow L, Astrup AS, Hovind P, Jacobsen PK, Alibegovic AC, et al. Effect of adjunct metformin treatment in patients with type-1 diabetes and persistent inadequate glycaemic control. A randomized study. PLoS ONE 2008;3:e3363.

[45] Moon RJ, Bascombe LA, Holt RI. The addition of metformin in type 1 diabetes improves insulin sensitivity, diabetic control, body composition and patient well-being. Diabetes Obes Metab 2007;9:143-5.

[46] Vella S, Buetow L, Royle P, Livingstone S, Colhoun HM, Petrie JR. The use of metformin in type 1 diabetes: a systematic review of efficacy. Diabetologia 2010;53:809-20.

[47] Lu H, Kraut D, Gerstenfeld LC, Graves DT. Diabetes interferes with the bone formation by affecting the expression of transcription factors that regulate osteoblast differentiation. Endocrinology 2003;144:346-52.

[48] Kume S, Kato S, Yamagishi S, Inagaki Y, Ueda S, Arima N, et al. Advanced glycation end-products attenuate human mesenchymal stem cells and prevent cognate differentiation into adipose tissue, cartilage, and bone. J Bone Miner Res 2005;20:1647-58.

[49] Stolzing A, Sellers D, Llewelyn O, Scutt A. Diabetes induced changes in rat mesenchymal stem cells. Cells Tissues Org 2010;191:453-65. 